\title{
Modelling Approach Used in the Real Time Emulator of the Rainbow Demonstrator
}

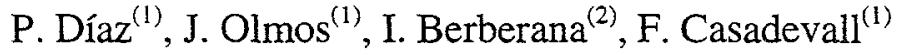 \\ (1) UPC, Campus Nord Mòdul D4 C/ Jordi Girona 1-3, 08034 Barcelona (Spain) \\ Phone: +34 3 4017201, Fax: +34 3 4017200, e-mail: pilar@xaloc.upc.es \\ (2) Telefónica I+D, C/ Emilio Vargas 4-6, 28043 Madrid (Spain) \\ Phone: +34 13374207 , Fax: +3413374352, e-mail: ibfm@tid.es
}

\begin{abstract}
A brief overview of the main characteristics of the Real Time Emulator (RTE) to be implemented in the context of the Rainbow project is presented in this paper. First of all, the RTE functional description is shown in order to justify the rationality of the envisaged approach. Next, a description of the emulation and simulation models used in the RTE for CoDiT is provided. Off-line simulation models are used to estimate the parameters to be employed in the emulation of the different scenarios, while the use of emulation models allows for a reduction of the RTE complexity.
\end{abstract}

\section{INTRODUCTION}

The basic objectives of the Radio Access Independent Broadband On Wireless (Rainbow) Project within the frame of the European Program ACTS are:

1. To demonstrate the feasibility and evaluate the complexity of a UMTS access infrastructure able to cope with different UMTS innovative radio access technique as well as representing a solution for the migration from second generation mobile systems.

2. To contribute to the UMTS standardisation process within ETSI and ITU.

3. To study possible solutions for the integration of the UMTS Radio Access System in the B-ISDN and IN context for both transport and control procedures.

4. To explore the impact of multimedia services and variable bit rate technique on the transport and control procedures of the UMTS Radio Access System.

The above objectives are pursued through the implementation of a demonstrator of the UMTS access infrastructure. One of the main objectives of RAP2 ${ }^{1}$ activities is the implementation of a Real Time Emulator,

\footnotetext{
${ }^{1}$ RAP2 is one of the workpackages of the Rainbow project devoted to define, implement and evaluate the emulated radio link based on the innovative radio interfaces, mainly CODiT and ATDMA.
}

which is part of such a demonstrator and emulates the lower layers of the innovative radio interfaces of a generic UMTS system, namely CODiT and ATDMA (in the RACE II context), to incorporate it in the RAINBOW demonstrator.

Two different types of models are required to characterize the behaviour of the different radio interfaces: simulation and emulation models. The simulation models are used to derive the behaviour, mainly in terms of error distribution, of the transport chains emulated in the RTE. The emulation models adopt a different approach, considering the system to emulate as a black box, the input/output behaviour of which intends to be reproduced without requiring a knowledge of the internal structure and processes. The internal structure of the model is normally not related to the internal structure of the real system or to its approximation in the simulation model.

The emulation models are used in the RTE mainly for two reasons: simulation models are too complex to be used in real time, and some functionalities, like coding and interleaving, will be implemented (not emulated). The use of emulation models implies a loss of accuracy with respect to simulation models (given that they take the inputs/outputs sequences provided by the simulation models). On the other hand, if they have been adequately selected, they allow the operation in real time with a moderate implementation complexity.

In the following sections, a description of the RTE architecture is first done. A simulation model required for the training of the emulation models for both the up and down-link is derived for CoDiT, and the parameters obtained from simulations and used in the emulator are explained.

\section{DESCRIPTION OF THE RTE}

The Real Time Emulator of the RAINBOW demonstrator is in charge of emulating the behaviour of the lower layers of the innovative radio interfaces in real time. The functional model adopted for the RTE consists of a Control Unit and some Information Processing Units, as shown in 
Figure 1. The Control Unit supports some mobility functions, including different types of handover and measurement reports. Data, voice and signaling messages exchanged between mobile terminal (MT) and base station (BTS) will pass through the Information Processing Unit. Each information unit is classified as requiring full integrity or not. Signaling messages belong to the former class, while speech information is an example of the latter case. The Information Processing Unit uses different approaches for each class of information. The type of processing that does not require integrity is referred to as Bit Oriented Processing (BOP), and the other is referred to as Packet Oriented Processing (POP).

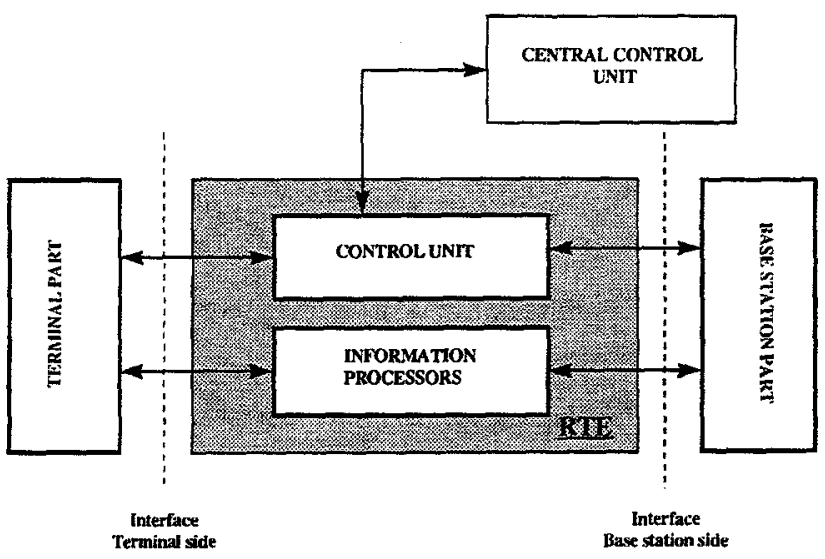

Figure 1.- Functional model for the Real Time Emulator.

The processing of the packet-oriented information is based on the concept of look-up tables. Packets that do not require retransmission are basically passed to upper layers tagged either as errored or not errored. Retransmission of packets is emulated by adding a certain amount of delay to each packet.

The bit oriented processing deals with the emulation of the transmission chain at bit level. The RTE must emulate in real time the input/output behavior of the chain shown in Figure 2.

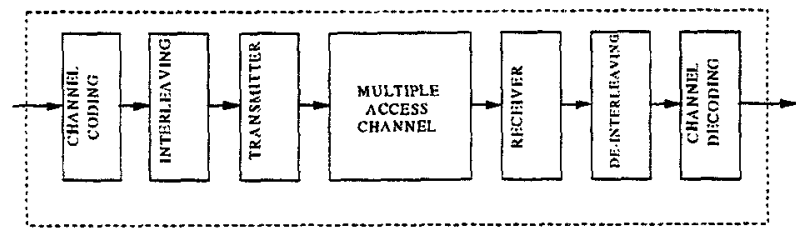

Figure 2.- Bit Oriented Processing (BOP): Emulated Transmission Chain.
For implementation of the BOP, two different parts are distinguished. The first part is the encoding system, which will be implemented by using standard available hardware. The second part, known as the inner system, includes the transmitter, channel and receiver (a RAKE receiver for CoDiT and an equalizer for ATDMA). Interleaving and de-interleaving may be incorporated to either part depending on the case. The inner part introduces errors in the transmitted bits in the same way as a real communication system. To model the inner part, Markov Chains based on the Hidden Markov Model (HMM) are used. For each testing scenario the Markov chains are properly trained through adequate off-line simulations. The main advantage of this approach is the reduction in time, resources and effort with regard to a real simulation of the inner system.

\section{CODIT SYSTEM LEVEL SIMULATIONS}

Link level models are required to characterize the short term error behaviour of the transmitter-channel-receiver chain for CoDiT.

\section{Simulation model for the PDCH}

As explained in [3], the CoDiT system uses two physical channels (PDCH for user data and PCCH for control data) for each user within a cell. These channels are transmitted simultaneously using two different spreading sequences. This applies for both the down and the up-link. Furthermore, in the down-link there exists another physical channel, the PICH (pilot channel), which is common to all the users of the cell and used for the estimation of the channel impulse response. The information bits in the PCCH are highly protected by robust channel codes, while the PICH channel is sent with $12 \mathrm{~dB}$ more energy than the PDCH of every user. For this reason it is assumed that the PICH channel is perfectly detected on the down-link, allowing perfect channel impulse response estimation, and that the $\mathrm{PCCH}$ is demodulated without errors, allowing perfect channel impulse response estimation on the up-link and correct knowledge of the logical channel multiplexing in the up and down-link. Therefore, the PCCH and the PICH (on the down-link) will only be considered as a source of cochannel interference.

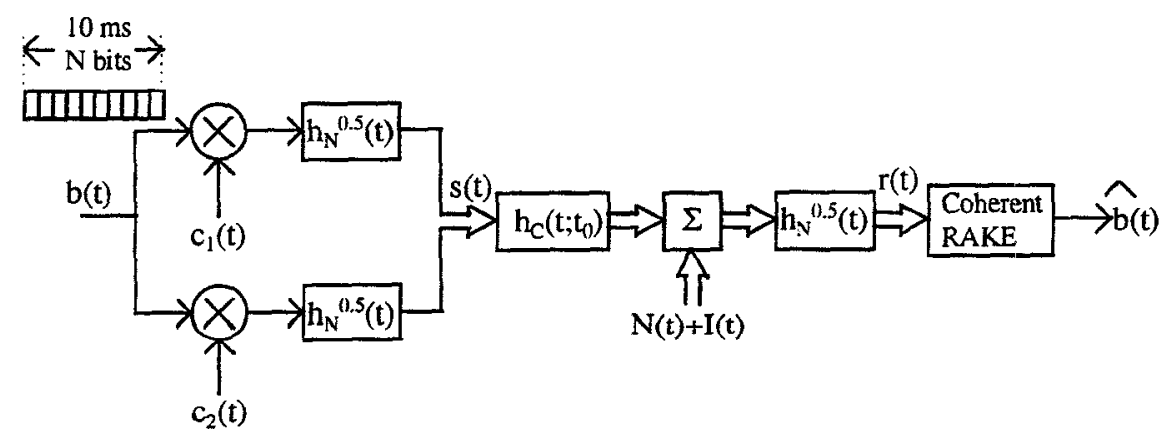

Figure 3.- System model for the PDCH transmission in the down-link. 


\section{Down-link system model for the PDCH}

Figure 3 is a block diagram of the low-pass equivalent system model for the PDCH transmission in the down-link. Since the logical contents of the frame are not relevant to model the system, the input signal, $b(t)$, is considered as a $10 \mathrm{~ms}$-long burst of rectangular shaped bit pulses. The number of bits in the frame, $N$, depends on the bit rate of that particular frame and is known by the receiver upon detection of the PCCH.

So, $b(t)$ can be written as:

$$
b(t)=\sum_{k=0}^{N-1} b_{k} \operatorname{rect}\left(t-k T_{b}\right)
$$

where $b_{k} \in\{+1,-1\}$ is the $k$-bit, $T_{b}$ is the bit period and $\operatorname{rect}_{h}(t)$ is a rectangular pulse with unit amplitude and $T$ second duration.

According to [3], the modulation in the down-link is QPSK at the chip level, so the input signal is simultaneously multiplied by the $\mathrm{PN}$ sequences $c_{7}(t)$ and $c_{2}(t)$, which are given by:

$$
\begin{aligned}
& c_{1}(t)=\sum_{n=-\infty}^{\infty} c_{n, 1} \delta\left(t-n T_{c}\right) \\
& c_{2}(t)=\sum_{n=-\infty}^{\infty} c_{n, 2} \delta\left(t-n T_{c}\right)
\end{aligned}
$$

where $c_{n, l}$ and $c_{n, 2}$ are, respectively, the chip values, $\delta(t)$ is the Dirac delta and $T_{c}$ is the chip period. In order to simplify the analysis, a complex notation is used for the spreading sequences:

$$
c(t)=\sum_{n=-\infty}^{\infty} c_{n} \delta\left(t-n T_{c}\right)
$$

with $c_{n}=c_{n, 1}+j c_{n, 2}$. After being adequately modulated by $b(t)$, the signal enters to the transmitter filter. The transmitter filter is a low-pass filter, the purpose of which is to spectrally shape the transmitted signal and, together with the receiver filter, to keep it free of interchip interference. In practice, a root raised cosine Nyquist filter, $\mathrm{h}_{\mathrm{N}}{ }^{0.5}(\mathrm{t})$, with a roll-off of 0.5 is used at both ends.

Therefore, the complex low-pass equivalent transmitted signal, $s(t)$, is given by:

$$
s(t)=b(t) \cdot c(t) * h_{N} 0.5(t)
$$

where * denotes convolution. The transmitted signal, $s(t)$, passes through the mobile channel, the low-pass equivalent signal of which is modeled as a complex time-variant linear filter with $\rho$ independent signal paths (WSSUS hypothesis). That is:

$$
h_{C}\left(t ; t_{0}\right)=\sum_{i=1}^{\rho} \alpha_{i}\left(t_{0}\right) \cdot \delta\left(t-\tau_{i}\right)
$$

where $\alpha_{i}\left(t_{0}\right)$ is the complex random variable modeling the propagation through the $i$-th scatter at time $t=t_{0}$ and $\tau_{i}$ is the propagation delay associated to that scatter. The delays, $\tau_{i}$, introduced by the different signal paths are assumed to be constant through all the simulation. The number of taps $(\rho)$, the statistics of $\alpha_{i}\left(t_{0}\right)$ and the values taken by $\tau_{i}$ $(i=1 . . \rho)$ are environment dependent and will be simulated according to the specifications given in [4]. However, it must be noted that only four branches are available at the CoDiT RAKE receiver.

The received signal, $r(t)$, can be written as,

$$
\begin{aligned}
r(t) & =\left(s(t) * h_{c}(t)+N(t)+I(t)\right) * h_{N} 0.5(t) \\
& =b(t) \cdot c(t) * h(t)+N_{f}(t)+I_{f}(t)
\end{aligned}
$$

where $N(t)$ models the front-end thermal noise and $I(t)$ models the co-channel interference. Notice that the filtered noise and interference have been defined as $N_{f}(t)$ and $I_{f}(t)$, respectively, and the overall channel impulse response as,

$$
h(t) \equiv h_{N} 0.5(t) * h_{c}(t) * h_{N} 0.5(t)=\sum_{i=1}^{\rho} \alpha_{i} \cdot h_{N}\left(t-\tau_{i}\right)
$$

It can be demonstrated that the decision variable at the output of a RAKE with $L$ branches is given by:

$$
\begin{aligned}
& v_{k}=b_{k} \cdot 2 g_{P D C H} \cdot \sum_{j=1}^{L}\left|h\left(t_{j}\right)\right|^{2}+\operatorname{Re}\left(\eta_{k}^{\prime}\right)+ \\
& +\sum_{j=1}^{L} \operatorname{Re}\left\{\begin{array}{l}
\left.\sum_{j=n_{j}}^{N_{j}} h^{*}\left(t_{j}\right) \cdot h\left(t_{j}-m T_{c}\right) \cdot R\left(m,\left\{b_{k}\right\}\right\}\right\} \\
(m \neq 0)
\end{array}\right\}
\end{aligned}
$$

where the first term is the useful part while the second and third terms respectively represent the thermal noise plus other users interference and the intersymbol interference. The parameter $\mathrm{g}_{\mathrm{PDCH}}=\mathrm{T}_{\mathrm{b}} / \mathrm{T}_{\mathrm{c}}$ is the spreading factor and the following definitions have also been considered:

$$
\begin{gathered}
R\left(m,\left\{b_{k}\right\}\right)=\sum_{l=0}^{g_{P D C H}-1} c_{1}^{*} c_{m+l} \cdot b\left((m+l) T_{c}\right) \\
E\left\{\left(\operatorname{Re}\left(\eta_{k}^{\prime}\right)\right)^{2}\right\}= \\
=\left(\sum_{j=1}^{L}\left|h\left(t_{j}\right)\right|^{2}+\frac{1}{2 N_{0}^{\prime}} \cdot \sum_{i=1 j=1}^{L} \sum_{(j \neq i)}^{L} R_{N_{f}^{\prime}}\left(t_{i}-t_{j}\right) \cdot h^{*}\left(t_{i}\right) \cdot h\left(t_{j}\right)\right. \\
R_{N_{f}^{\prime}}\left(t_{i}-t_{j}\right)=2 N_{0} \cdot h_{N^{\prime}}\left(t_{i}-t_{j}\right) \\
2 N_{0}^{\prime}=2 N_{0}+I_{1}+I_{2}
\end{gathered}
$$

The first term $2 N_{0}$ models thermal noise, while $I_{1}$ and $I_{2}$ respectively model intra-cell and inter-cell interference.

\subsubsection{Simulation model of the PDCH for the down-link.}

Once the expression for the decision variable at the output of the RAKE is known, it is possible to develop a simulation model to train the Markov chain. From the $v_{k}$ expression, it is obvious that a mobile channel simulator is needed. A complex, time-variant FIR filter with impulse response $h\left(t ; t_{0}\right)$ has been considered. Concerning the simulation model of the PDCH, Figure 4 shows the global block diagram of it. 


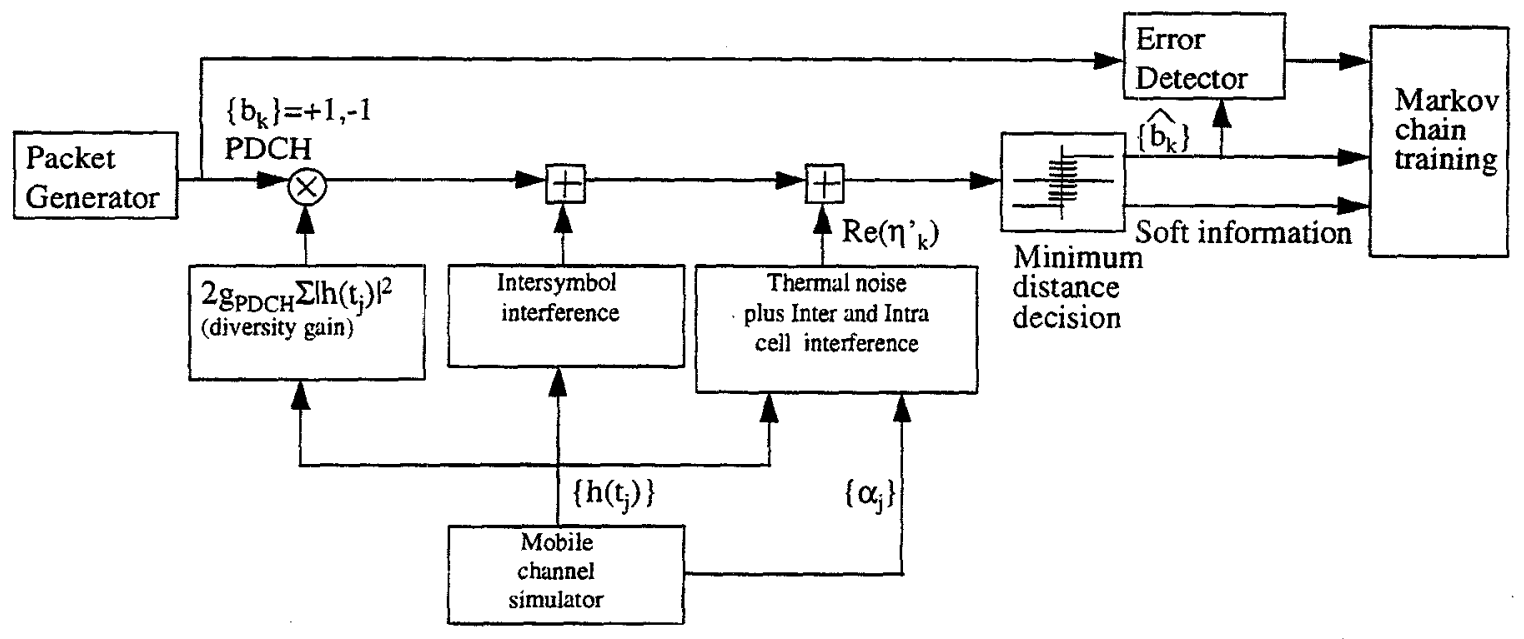

Figure 4.- Block diagram of the simulation model for the PDCH on the down-link.

The bits of the PDCH are randomly generated, and for every bit, the RAKE output is computed. The noise is simulated by means of a gaussian random variable, the variance of which depends on the current mobile channel impulse response and changes from bit to bit.

Finally, the decision variable is compared to zero to decide whether a logical ' 0 ' or ' 1 ' has been transmitted. The detected packet of bits, as well as the soft-information (in the form of distance to the threshold) and the obtained error pattern will be used to train the Markov chain.

\subsubsection{Up-link system model for the PDCH.}

The CoDiT system concept for the uplink differs from the downlink concept in some aspects. The first one is the absence of PICH channel to provide channel impulse response estimation. In turn, the uplink channel impulse response is estimated from the $\mathrm{PCCH}$. This makes possible the demodulation of the PDCH using a coherent RAKE receiver of four branches (as in the downlink).

Another feature of the uplink is the use of a power control loop to guarantee that, regardless of the distance, the basestation will receive a fixed signal level. As the channel may show high Doppler spread, only mean power control is possible. In our simulations it is assumed perfect mean power control. This means that all the mobiles within a cell reach to the base-station with the same mean signal power.

By considering these assumptions the system model for the uplink is essentially the same that the already proposed for the downlink. In fact, the block diagram of the uplink model is equal to the one shown in Figure 3. Furthermore, taking only into account the wanted signal plus thermal noise the decision variable will have the same expression that the already calculated for the downlink. Only the cochannel interference requires a different treatment.

With respect to the co-channel interference, a distinction between intra-cell and inter-cell interference must be made. The simulation approach proposed is to use gaussian noise to simulate both kinds of interference. As in the downlink, only the central portion of the interfering spectral density is of interest. The spectral density of the interfering signals is calculated by modeling them as random sequences, and if it is assumed that all the sequences are pairwise uncorrelated, then their variances can be added. It is proposed to keep constant, and equal to its mean value, the interference power received from each active user within the cell, and with respect to the inter-cell interference it is proposed to keep its power variable and equal to a certain percentage of the total intra-cell interference power. The simulation model for the uplink is the same that the one used for the downlink with the only difference that the interfering noise must be accordingly calculated.

\section{MARKOV MODELS OF THE RADIO CHANNEL}

Once the statistical behavior of the radio channel (in terms of error distribution) has been characterized by means of the simulations, it is necessary to reproduce this statistical behavior in the RTE. The selected technique to do this in the RTE is the Hidden Markov Model.

When a channel model is based on Markov chains, there is a correlation between consecutive blocks, so the number of errors within a transmitted block only depends on the number of the previous block. Therefore, the states of the Markov chain are given by the number of errors within a block

To avoid having as many states as bits in a block, it is more convenient to a-priori establish the number of states, $N$. In this case, each state represents a range of variation of error number, and they must be as equiprobable as possible.

The file containing the statistics parameters for the Markov chain must contain:

1. A block error histogram in order to specify for each state the minimum and the maximum number of errors as well as the error number mean.

2. An NxS matrix containing the mean soft value per burst. 
3. An SxS matrix for each state and for correct bits containing the $p d f$ (a kind of probability density function).

4. An SxS matrix for each state and for erroneous bits containing the $p d f$ (a kind of probability density function).

5. A transition matrix in order to determine the transition probability from state $i$ to state $j$ in the Markov chain.

Validation of the model is currently being carried out for a relatively low number of states $(\mathrm{N}=4$ and $\mathrm{N}=8)$. The validation procedure is based on the comparison of five statistic parameters obtained from both link level simulation results and Hidden Markov Model results. These parameters are the error free interval distribution, the error cluster distribution, the probability that a block of $\mathrm{m}$ bits contains exactly $\mathrm{k}$ errors, the probability of an error burst of length $k$ in a block of length $m$ and the normalised covariance function.

\section{CONCLUSIONS}

In this paper, the link-level simulation models to be used for deriving the data required in the real time emulation process of CoDiT have been described and analyzed. These models are being used to generate the information required to derive the parameters of the emulation models used in the RTE. These emulation models are based on the concept of the Hidden Markov Model, the parameters of which have also been described. Future work will deal with the validation of this concept to be used in the RTE for both CODiT and ATDMA.

\section{ACKNOWLEDGMENTS}

This work is part of the Rainbow project (AC015), partially funded by the European Commission under the Advanced Communications Technologies and Services (ACTS) framework.

\section{REFERENCES}

[1] L.R. Rabiner, B.H. Juang, "An Introduction to Hidden Markov Models", IEEE ASSP Magazine, Vol.3, January 1986.

[2] F. Swarts, H.C. Ferreira, "Markov Characterisation of Channel with Soft Decision Outputs", IEEE Transactions on Communications, Vol. 41, n5, May 1993.

[3] CEC Document CODIT/ERA/PM/DS/P/050/b1 "CODIT Final Review Report".

[4] CEC Document MPLA/TDE/SIG5/DS/P/001/b1, "Common Basis for Evaluation of ATDMA and CODIT System Concepts", M. Pizarroso, J. Jiménez (ed.). 